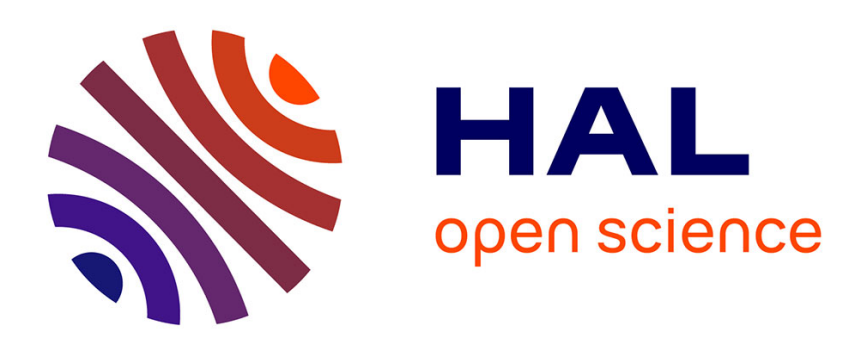

\title{
Efficiency of the Bio-Inspired Leaky Integrate-and-Fire Neuron for Signal Coding
}

Effrosyni Doutsi, Lionel Fillatre, Marc Antonini

\section{To cite this version:}

Effrosyni Doutsi, Lionel Fillatre, Marc Antonini. Efficiency of the Bio-Inspired Leaky Integrate-andFire Neuron for Signal Coding. EUSIPCO (27th European Signal Processing Conference), Sep 2019, Coruña, Spain. hal-02146548

\section{HAL Id: hal-02146548 https://hal.science/hal-02146548}

Submitted on 8 Jan 2021

HAL is a multi-disciplinary open access archive for the deposit and dissemination of scientific research documents, whether they are published or not. The documents may come from teaching and research institutions in France or abroad, or from public or private research centers.
L'archive ouverte pluridisciplinaire HAL, est destinée au dépôt et à la diffusion de documents scientifiques de niveau recherche, publiés ou non, émanant des établissements d'enseignement et de recherche français ou étrangers, des laboratoires publics ou privés. 


\section{Efficiency of the bio-inspired Leaky Integrate-and-Fire neuron for signal coding}

\author{
Effrosyni Doutsi \\ Institute of Computer Science \\ Foundation for Research \& Technology-Hellas \\ Heraklion, Greece \\ edoutsi@ics.forth.gr
}

\author{
Lionel Fillatre \\ Université Côte d'Azur \\ CNRS \\ I3S laboratory \\ lionel.fillatre@i3s.unice.fr
}

\author{
Marc Antonini \\ Université Côte d'Azur \\ CNRS \\ I3S laboratory \\ am@i3s.unice.fr
}

\begin{abstract}
The goal of this paper is to investigate whether purely neuro-mimetic architectures are more efficient for signal compression than architectures that combine neuroscience and state-of-the-art models. We are motivated to produce spikes, using the LIF model, in order to compress images. Seeking solutions to improve the efficiency of the LIF in terms of the memory cost, we compare two different quantization approaches; the Neuroinspired Quantization (NQ) and the Conventional Quantization (CQ). We present that when the LIF model and the NQ appear in the same architecture, the performance of the compression system is higher compared to an architecture that consists of the LIF model and the CQ. The main reason of this occurrence is the dynamic properties embedded in the neuro-mimetic models. As a consequence, we first study which are the dynamic properties of the recently released (NQ) which is an intuitive way of counting the number of spikes. Moreover, we show that some parameters of the NQ (i.e. the observation window and the resistance) strongly influence its behavior that ranges from non-uniform to uniform. As a result, the NQ is more flexible than the CQ when it is applied to real data while for the same bit rate it ensures higher reconstruction quality.
\end{abstract}

Index Terms-Neuro-inspired quantization, uniform scalar quantizer, Leaky Integrate-and-Fire (LIF), spikes, entropy.

\section{INTRODUCTION}

Over the last few years, a lot of efforts have been devoted to the visual perception. While these efforts were initially focused on the understanding of the visual system as a quality assessment metric that perceives the visual stimulus, their scope has been widened today trying to mimic the processing mechanisms of the visual system in order to build bioinspired coding/decoding algorithms. These new algorithms are expected to provide qualitative results which are pleasant to the human eyes. In addition, the characteristic properties of the visual system such as the dynamic processing of the visual stimulus, its plasticity and its ability to generate a very sparse but informative code of spikes [1] seems to be beneficial to the progress of the state-of-the-art compression algorithms which are currently based on computationally greedy mechanisms like motion estimation [2].

The neurons are considered to be one of the most important processing units of the visual system. If a neuron is stimulated by a strong input, it will generate a sequence of electrical impulses which are called spikes, otherwise it will remain silent. The main concern when one aims to encode an image into spikes is if a spike train is informative enough to enable high quality reconstruction. If yes, then, what is the memory cost to store this information? It has been shown that some Spike Interpretation Mechanisms (SIM), like the Leaky Integrateand-Fire (LIF) [1], [3] model or the Rank Order Coding (ROC) [4], [5] among others, are able to interpret a sequence of spikes focusing on the time or the rank the first spike of each neuron within a population arises. Encoding this information is sufficient to recover high quality images.

It has been proven in [6] that for a given input, $I$, if we encode the arrival delay of the first spike, $d$, the LIF model enables to perfectly recover every input stimulus which had previously excited a neuron but at a very high memory cost. To reduce the memory cost it was proposed in [7] the Neuroinspired Quantization (NQ); an intuitive and simple solution of counting the number of spikes. In that sense, a population of some neurons that are not all excited at the same time, but have emitted the same number of spikes, will be grouped together. The encoding architecture consisting of the LIF and the NQ was compared to the Uniform Dead-zone Quantizer (UDQ) when the aforementioned is applied to the pixel intensities of an image providing very promising results [7].

In this paper, we are challenging whether the "dynamic" way of counting the spikes is the most efficient way to reduce the memory cost of the LIF [6] or the "static" signal processing tools such as the state-of-the-art Uniform Scalar Quantizer (USQ) [8] is more appropriate to improve the LIF's performance. To reach this goal, we first study the properties of the NQ [7] and how strong is the impact of some of its parameters on NQ's performance. We prove later on that, depending on the parameters, the behavior of the NQ ranges from non-uniform to uniform. In addition, we show at the experimental results that the NQ could be much more efficient that CQ in improving the performance of LIF.

Section II is a brief recall of the neuro-inspired quantizer. Section III is a discussion concerning the dynamic properties of the NQ with respect to its parameters. In section IV we introduce the USQ as an alternative architecture of how to improve the memory efficiency of the perfect-LIF, called Conventional Quantization (CQ) of the delays. Section V is dedicated to the comparison between NQ and CQ while in section VI we draw some conclusions of this work. 


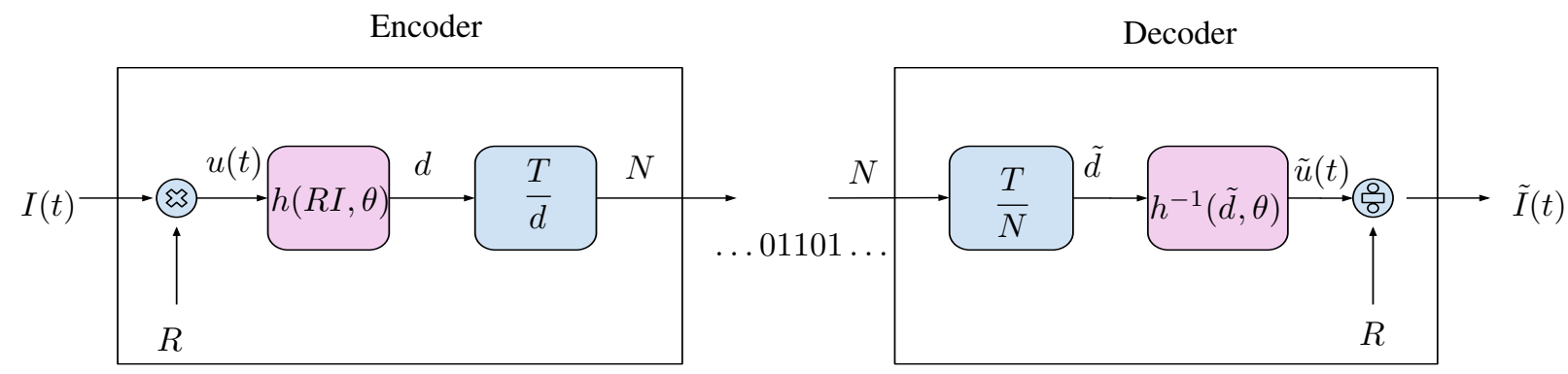

Fig. 1. This coding/decoding architecture combines the perfect-LIF model (pink) with the neuro-inspired quantizer (blue) introduced in [7] where, $d$ is the delay of the first spike, $T$ is the observation duration and $N$ the number of emitted spikes

\section{Recall of the Neuro-inspired Quantization}

The LIF model approximates a neuron by an electrical circuit [1]. Under the assumption that the input signal is constant during some time $T, I(t)=I \mathbf{1}_{[0 \leq t \leq T]}(t)$, each $I$ can be described by the arrival delay $d$ of the first spike according to:

$$
d= \begin{cases}+\infty, & R I<\theta, \\ h(R I ; \theta)=-\tau_{m} \ln \left[1-\frac{\theta}{R I}\right], & R I>\theta,\end{cases}
$$

where $R$ is the resistance, $\tau_{m}=R C$ is the leaky integrator term, $C$ is the capacitor of the electrical circuit and $\theta$ is the membrane threshold of the neuron. It was introduced in [6] that if the delay $d$ is known at the decoder, using the $h^{-1}(d ; \theta)$ function it is possible to perfectly reconstruct every input intensity which is higher than the threshold while $d \in(0, T]$ :

$$
\tilde{I}= \begin{cases}0, & d>T, \\ I=h^{-1}(d ; \theta), & d \leq T .\end{cases}
$$

Due to the high memory cost of the perfect-LIF [6], the NQ was introduced in [7] where it is proven that for recovering the input signal, it is efficient enough to encode the number of spikes $N$ emitted during some time $T$, instead of the delays, (see Fig. 1):

$$
N= \begin{cases}0, & d>T, \\ \left\lfloor\frac{T}{d}\right\rfloor, & d \leq T .\end{cases}
$$

In this case, it is possible to approximate the spike arrival delays $\tilde{d}=T / N$ and reconstruct the best possible values:

$$
\tilde{I}= \begin{cases}0, & N=0, \\ h^{-1}(\tilde{d} ; \theta)=h^{-1}\left(\frac{T}{N} ; \theta\right), & N \neq 0 .\end{cases}
$$

\section{DyNAMIC PRoperties of THE NEURO-INSPIRED QUANTIZATION}

\section{A. Observation Window}

The "dynamic" behavior of the NQ is one of its most important properties associated with the fact that the number of spikes depends on the length of the observation window
$T$. According to (3), the longer the input image is flashed, the more the spikes that correspond to each input intensity. On the other hand, if the observation window is too small, the number of spikes will fail to precisely describe the input signal. Figure 2 (a) shows for different $R$ values that while increasing the observation window $T$, the quality of the reconstructed signal substantially improves.

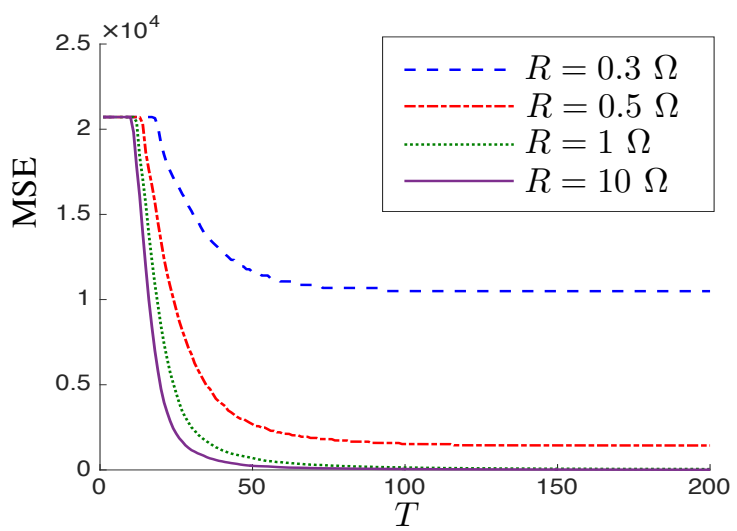

Fig. 2. This figure illustrates the impact of the size of the observation window $T$ on the performance of the $N Q$. The reconstruction quality improves when the size of the observation window increases (set of parameters: $C=50 \mathrm{~F}$, $\theta=50)$.

\section{B. Resistance}

Another parameter that substantially influences the behavior of the NQ is the resistance $R$. It has been shown in [7] that the NQ can be approximated by a USQ for very large values of $R$. In this work, we extend that proof and we show that $R$ determines the behavior of NQ either for small or large values.

Proposition 1. Let us assume that the input value I has generated exactly $k$ spikes for any $k \geq 1$ and the $R$ is arbitrarily large. Then, the $N Q$ is a uniform quantizer where the length $\ell_{k}$ of each quantization interval is constant for all $k$

$$
\ell_{k}=\frac{\theta C}{T}+o\left(\frac{1}{R}\right), \quad \forall k,
$$

where the notation $o(\cdot)$ is the little-o notation which is used to express the asymptotic behavior of a function. 
Proof. The proof, which is based on Taylor series, has been omitted due to the lack of space.

Proposition 2. Let us now assume that $R$ is small, then the $N Q$ is a non-uniform quantizer. The length of each quantization interval depends on the number of spikes $k$. When $k$ increases, the length $\ell_{k}$ converges to an asymptotic value

$$
\ell_{k}= \begin{cases}h^{-1}(T), & k=0, \\ h^{-1}\left(\frac{T}{k+1}\right)-h^{-1}\left(\frac{T}{k}\right), & k>0, \\ \frac{\theta C}{T}, & k \rightarrow \infty .\end{cases}
$$

Proof. The proof, which is based on Taylor series, has been omitted due to the lack of space.

Figure 3 illustrates how the value of $R$ affects the length $\ell$ of the quantization intervals in function of the number of spikes. It is obvious that when $R$ is small, the length of the quantization interval varies with respect to the number of spikes $k$ until it converges to the asymptotic case. However, when $R$ is large, the NQ becomes completely uniform. As we show later on, when the NQ is applied to a normal distribution signal it is expected to better encode the low intensities than the high intensities when $R$ is small. On the contrary, whatever the intensity is if $R$ is high it will be treated in a uniform manner. The interpretation of the above behavior will be more evident in the section $\mathrm{V}$.

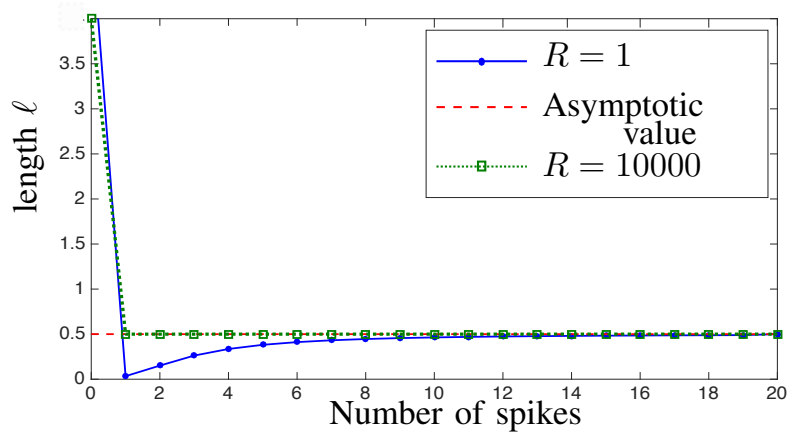

Fig. 3. This figure illustrates the performance of the NQ as a non-uniform quanizer when the value of $R$ is small and as a uniform quantizer when the value of $R$ is arbitrarily large (set of parameters: $\theta=5, C=10 \mathrm{~F}$ and $T=100 \mathrm{~ms}$.

\section{Conventional Quantization}

In this section, we propose an alternative architecture based on conventional signal processing techniques in order to improve the performance of the perfect-LIF with respect to the memory cost. We propose to assign the delay values into a smaller finite group using as a conventional quantization (CQ) the Uniform Scalar Quantizer (USQ):

$$
Q_{q}(d)= \begin{cases}+\infty & d=+\infty \\ \left(\left\lfloor\frac{d}{q}\right\rfloor+\frac{1}{2}\right) \times q, & \text { otherwise }\end{cases}
$$

where $q$ is the quantization step which defines the size of the quantization layers. If $q$ is large, the distortion of the reconstructed image will be high. On the contrary, if $q$ is small the size of the quantization layers will be small, thus the quality of the reconstructed image will be high.

Throughout this work, the CQ is said to be "static", meaning that its performance will be the same even if the size of the observation window $T$ varies. This is absolutely coherent to the definition of the LIF model (1) where the first spikes appears always with the same delay determined by the input intensity $I$ and the threshold $\theta$. However, according to (4) this is not the case for the NQ that strongly depends on the size of the observation window. Figure 4 shows the complete architecture of this "hybrid" coding/decoding system that consists of the LIF model and the USQ. For a given input $I$ we apply the perfect-LIF encoder in order to compute the arrival delay $d$ of the first spike. To reduce the redundancy of the delay values, we apply the CQ to obtain the quantized delays $\hat{d}$. The perfect-LIF decoder receives $\hat{d}$ and reconstructs an approximation of the input $\tilde{I}$.

\section{EXPERIMENTAL RESULTS}

This section aims to compare whether the performance of the perfect-LIF encoder/decoder is improved by the use of the NQ or the CQ. Let $I_{1}, \ldots, I_{n}$ be the pixel intensities of an image with a size $n=256 \times 256$. Each neuron receives an input intensity which is characterized by the arrival delay of the first spike according to (1). Each arrival delay is quantized by the NQ (3) and the CQ (7).

Figure 5 compares the rate-distortion curves of the NQ and the CQ when the observation window $T$ is fixed. For this experiment we tested 100 natural images of the size $n=256 \times$ 256 taken from the USC-SIPI database [9]. This plot presents the average response of these images. On the one hand, we test the performance of the NQ for different threshold values $\theta$. On the other hand, for a given threshold value we tested the performance of the CQ for different quantization steps $q$. We used two qualitative metrics, the Peak Signal to Noise Ratio (PSNR) (see Fig. 5 (a)) and the Structure SIMilarity (SSIM) [10] (see Fig. 5 (b)). The rate was computed by the Shannon Entropy as following:

$$
H=-\sum_{i=} p_{i} \log _{2} p_{i},
$$

where $p_{i}$ is the probability mass function of each different possible number of spikes $N_{i}$ for the NQ or each different possible quantized delay $\hat{d}_{i}$ for the CQ. Throughout this paper the entropy is given in bits per pixel (bpp).

Figure 6 illustrates the reconstruction results of an image encoded by the perfect-LIF and (i) the NQ or (ii) the CQ for similar bit rates. As a consequence of the theoretical study given in section III, we have chosen a very small ( $T=10 \mathrm{~ms}$ ) and a sufficiently large $(T=100 \mathrm{~ms})$ observation window to convince the reader about the impact of this parameter on the performance of the NQ when the rest of the parameters are fixed. It is obvious that the degradation of the reconstructed 
Encoder

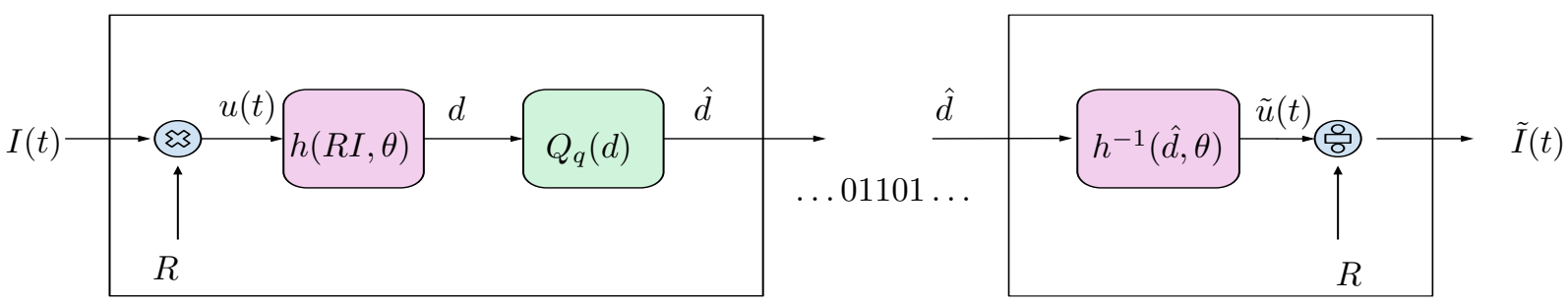

Fig. 4. This coding/decoding architecture combines the perfect-LIF model (pink) with the conventional quantizer (green).
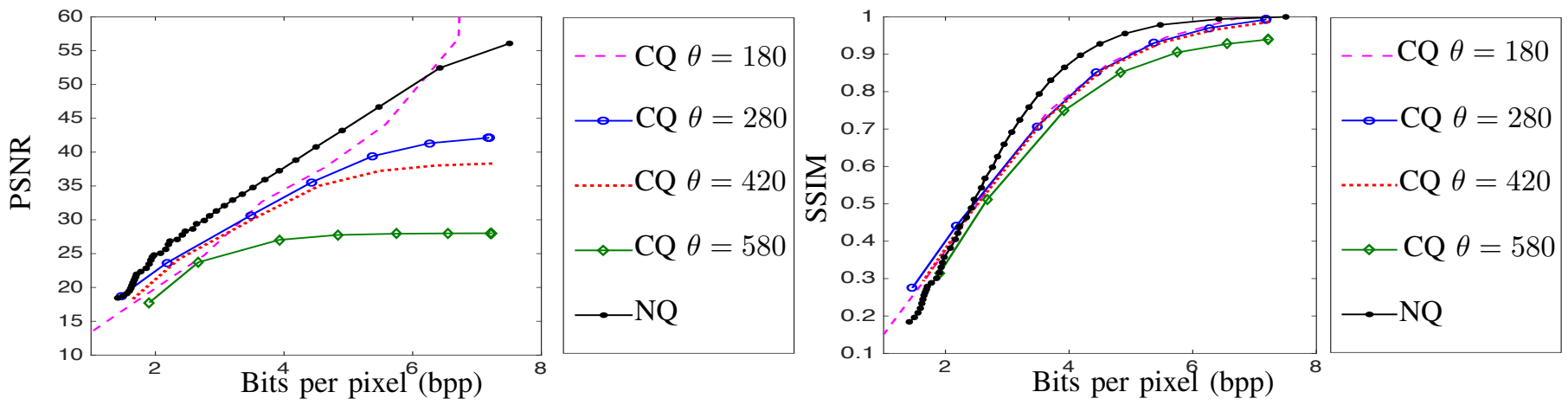

Fig. 5. This figure illustrates the average responses of 100 natural images which have been tested to compare the performance of the NQ (black solid-square line) and the CQ (colorful curves). We obtained the NQ for different $\theta$ values when $T$ is fixed. The CQ curves present the behavior of the quantizer for different $q$ values when $\theta$ and $T$ are fixed. According to this plot, the NQ outperforms CQ. (a) rate vs MSE (b) rate vs SSIM. (Set of parameters: $\theta \in$ [1, 700] $q=\{0.001,0.01,0.1,0.5,1,2,4,10,20\}, T=10 \mathrm{~ms}, C=10 \mathrm{~F}, R=1000 \Omega$.)

image is high (e)-(h) when $T$ is small, because the spikes are not enough to enhance the visual quality of the reconstructed image. However, when $T$ is large, the distortion of the signal is small (a)-(d). Comparing NQ with CQ it is crystal clear that for similar bit rates the "dynamic" NQ outperforms the "static" CQ.

\section{CONCLUSION}

In this work, we have studied the dynamic properties of the recently released Neuro-inspired Quantization (NQ) and we have shown that this pure neuro-mimetic and "dynamic" model is much more efficient than the "static" conventional signal processing tools. To achieve this goal, we first proposed an analysis of the dynamic behavior of the NQ that ranges from a non-uniform to a uniform one depending on the parameters' setting. In addition, we have shown that the NQ is much more efficient that the uniform scalar quantizer, which is a conventional quantization method when they are chosen to reduce the memory cost of the LIF model. These results come to enhance the investigation of the neuro-inspired coding/decoding architecture which seems to be very promising and flexible to obtain a better reconstruction comparing to the state-of-the-art.

Last but not least, there are several open issues and extensions concerning this work. First of all, we are interested in building a complete neuro-mimetic coding/decoding system and compare its performance to the state-of-the-art compression standards like JPEG and JPEG2000. In addition, we believe that the dynamic properties of NQ would have a significant impact on time-varying inputs such as video streams. However, this extension is very challenging as it requires a cautious design of the spike generation in order to preserve the semantic information along time.

\section{REFERENCES}

[1] W. Gerstner and W. Kistler, Spiking neuron models: Single Neurons, Populations, Plasticity, Cambridge University Press, 2002.

[2] C. Stiller and J. Konrad, "Estimation motion in image sequences," IEEE Signal Processing Magazine, vol. 16, no. 4, pp. 70-91, 1999.

[3] R. B. Stein, "Some Models of Neuronal Variability," Biophysical Journal, vol. 7, pp. 37-68, 1967.

[4] S. J. Thorpe and J. Gautrais, "Rank Order Coding: A new coding scheme for rapid processing in neural network," Computational Neuroscience: Trends in Research, pp. 113-118, 1998.

[5] R. VanRullen and S. J. Thorpe, "Rate coding versus temporal order coding: what the retinal ganglion cells tell the visual cortex," Neural Computation, vol. 13, no. 6, pp. 1255-1283, 2001.

[6] E. Doutsi, L. Fillatre, M. Antonini, and J. Gaulmin, "Bio-inspired Sparse Representation of Images," in Groupe d'Etudes du Traitement du Signal et des Images (Gretsi), 2017.

[7] E. Doutsi, L. Fillatre, M. Antonini, and J. Gaulmin, "Neuro-inspired Quantization," in 2018 25th IEEE International Conference on Image Processing (ICIP), Athens, Oct 2018, pp. 689-693.

[8] R. M. Gray and D. L. Neuhoff, "Quantization," IEEE Transactions on Information Theory, vol. 44, no. 6, pp. 2325-2384, 1998.

[9] A. Weber, "The USC-SIPI Image Database," 1977.

[10] Zhou Wang, A C Bovik, H R Sheikh, Student Senior Member, E. P. Simoncelli, and Student Senior Member, "Image quality assessment: From error visibility to structural similarity," IEEE Transactions on Image Processing, vol. 13, no. 4, pp. 600-612, 2004. 


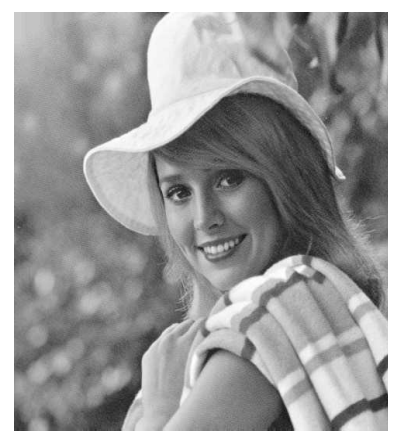

(a) Original

Input

Image

$n=256 \times 256$ pixels

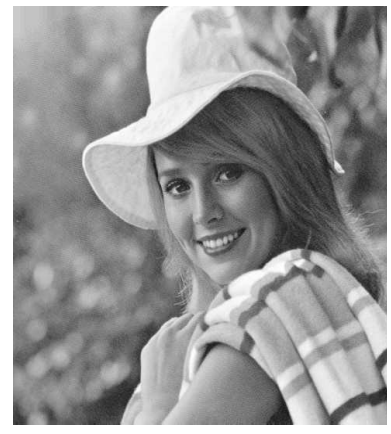

(e) NQ

$H=7.5 \mathrm{bpp}$

$\mathrm{PSNR}=54.16 \mathrm{~dB}$

$\theta=1, T=10 \mathrm{~ms}$

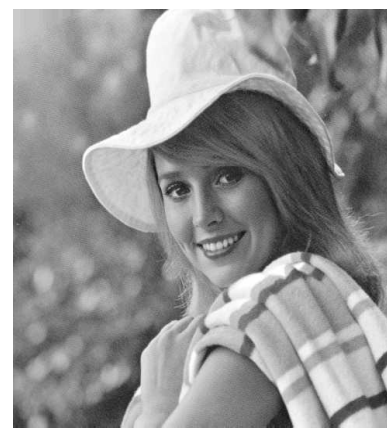

(i) $\mathrm{CQ}$

$H=6.43 \mathrm{bpp}$

PSNR $=55.37 \mathrm{~dB}$

$\theta=161, q=0.1$

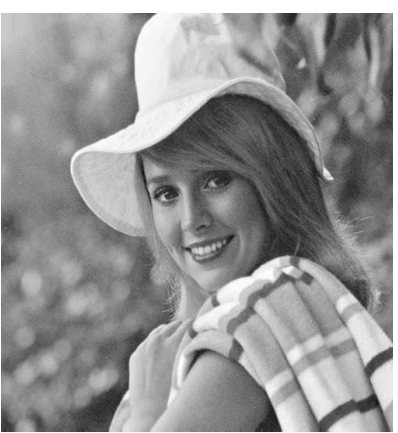

(b) NQ

$H=6.42$ bpp

PSNR $=52.45 \mathrm{~dB}$

$\theta=20, T=100 \mathrm{~ms}$

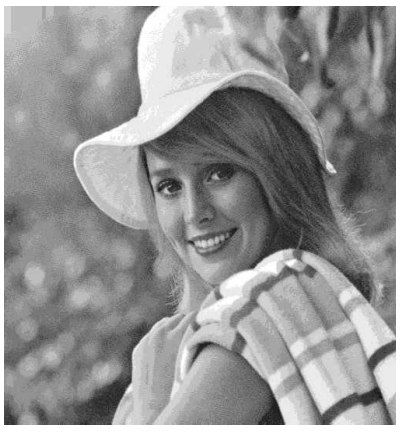

(f) NQ

$H=3.15$ bpp

PSNR $=32.37 \mathrm{~dB}$

$\theta=20, T=10 \mathrm{~ms}$

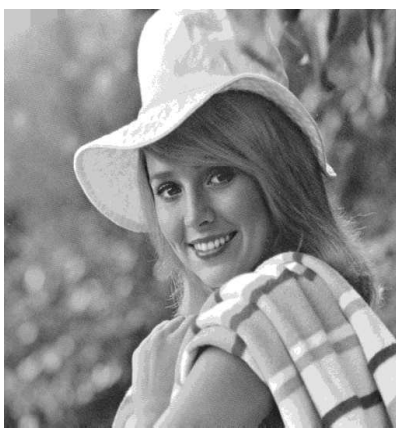

(j) $\mathrm{CQ}$

$H=4.37 \mathrm{bpp}$

$\mathrm{PSNR}=36.6 \mathrm{~dB}$

$\theta=180, q=1$

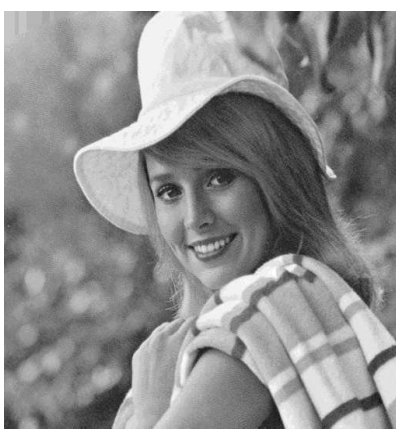

(c) NQ

$H=3.7 \mathrm{bpp}$

PSNR $=35.93 \mathrm{~dB}$

$\theta=140, T=100 \mathrm{~ms}$

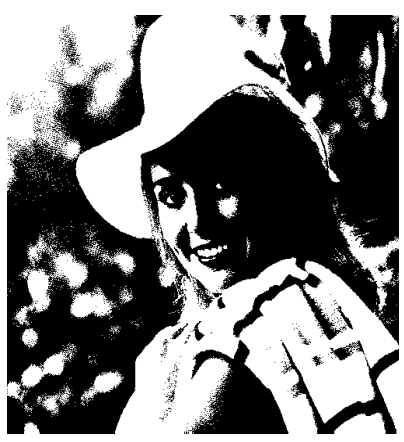

(g) NQ

$H=0.99$ bpp

$\mathrm{PSNR}=9.67 \mathrm{~dB}$

$\theta=140, T=10 \mathrm{~ms}$

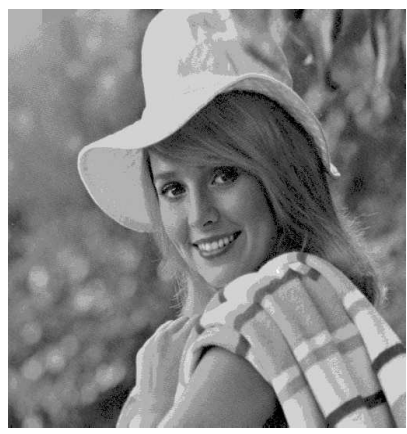

(k) CQ

$H=3.7 \mathrm{bpp}$

PSNR $=30.95 \mathrm{~dB}$

$\theta=480, q=4$

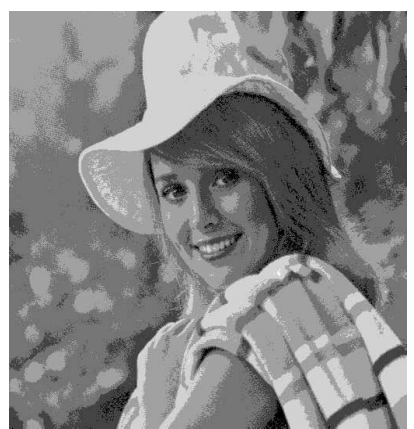

(d) NQ

$H=1.97 \mathrm{bpp}$

PSNR $=24.78 \mathrm{~dB}$

$\theta=500, T=100 \mathrm{~ms}$

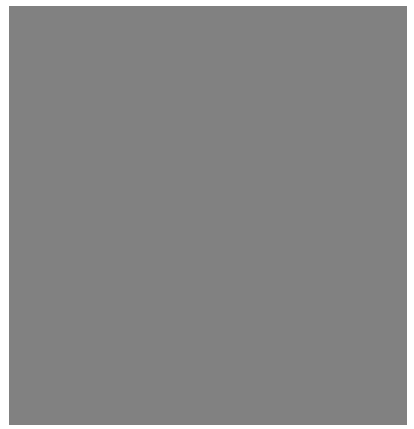

(h) NQ

$H=0$ bpp

PSNR $=4.96 \mathrm{~dB}$

$\theta=500, T=10 \mathrm{~ms}$

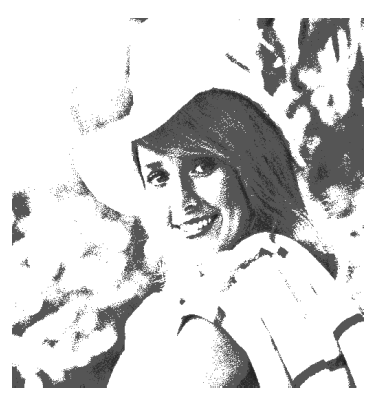

(1) $\mathrm{CQ}$

$H=1.98$ bpp

PSNR $=18.51 \mathrm{~dB}$

$\theta=61, q=2$

Fig. 6. This figure compares visually the performance of NQ and CQ when they are applied to the delays. When the observation window is large enough $T=100 \mathrm{~ms}$ (a)-(d), the NQ outperforms the CQ (i)-(l). However, if the observation window is small (e)-(h), the performance of NQ is limited whereas CQ's behavior remains constant (set of parameters: $R=1000 \Omega, C=10 \mathrm{~F}$ ). 